\title{
1 Comparación de tres modelos de etiquetado nutricional frontal de productos industrializados en Perú, 2019
}

DOI: 10.17533/udea.penh.v22n1a03

PERSPECTIVAS EN NUTRICIÓN HUMANA

ISSN 0124-4108

Escuela de Nutrición y Dietética, Universidad de Antioquia. Medellín, Colombia

Vol. 22, N. ${ }^{\circ}$, enero-junio de 2020, pp. 35-45.

Artículo recibido: 2 de octubre de 2019

Aprobado: 24 de julio de 2020

Vannya Inés Pineda Soto ${ }^{1 *}$; Ela Leila del Socorro Estrada Oré2

- - Resumen

Antecedentes: el etiquetado nutricional frontal es una herramienta para orientar la elección del consumidor hacia una dieta equilibrada y sana. Objetivo: conocer el modelo de etiquetado nutricional frontal que más influye en la identificación de calorías y nutrientes críticos de productos industrializados en adultos de Lima, Perú. Materiales y métodos: estudio cuasiexperimental-transversal en el que participaron 384 adultos de ambos sexos distribuidos en cuatro grupos equitativos: sin modelo de etiquetado nutricional, Guía Diaria de Alimentación, etiquetado de advertencia octogonal y Guía Diaria de Alimentación-semáforo. A cada participante se le aplicó un cuestionario con 10 figuras de empaques con el modelo de etiquetado, según el grupo al que correspondían, y 10 preguntas, con un puntaje total de 0 al 20 , según el número de respuestas positivas en la identificación de calorías y nutrientes críticos. Resultados: los mayores puntajes totales fueron para los

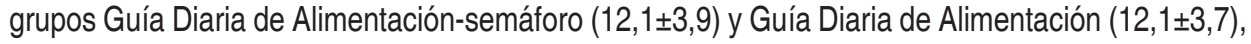
con diferencias significativas respecto a los otros dos grupos. También se presentaron diferencias en los puntajes de la identificación de calorías, grasas saturadas y grasa total; los valores más altos fueron para los dos grupos señalados $(p<0,05)$. No se observaron diferencias en la identificación de azúcar y sodio. Conclusiones: Ios modelos Guía Diaria de Alimentación y Guía Diaria de Alimentación-semáforo son los que más influyen en la identificación de calorías y nutrientes críticos.

Palabras clave: etiquetado de alimentos, etiquetado de productos, etiquetado nutricional, valor nutritivo.

$1^{*}$ Autor de correspondencia. Maestra en Nutrición y Dietética. Universidad Femenina del Sagrado Corazón. Lima, Perú. Orcid: https://orcid.org/0000-0001-9031-9119. Correo electrónico: vpineda.nutricion@ gmail.com

2 M. Sc. Food Engineering University of Massachusetts, EE. UU. Doutora en Engenharia de Alimentos Universidade de Campinas, Brasil. Orcid: https://orcid.org/0000-0002-0821-5810. Correo electrónico: leilaestradao@unife.edu.pe

Cómo citar este artículo: Pineda Soto Vl; Estrada Oré EL. Comparación de tres modelos de etiquetado nutricional frontal de productos industrializados en Perú, 2019. Perspect Nutr Humana. 2020;22:35-45. DOI: 10.17533/udea.penh.v22n1a03 


\section{Comparison of Three Types of Nutrition Label on Processed Food Packages in Peru, 2019}

\section{Abstract}

Background: A front-of-package nutrition label is a tool to help guide food selection towards a balanced and healthy diet. Objective: To understand the model of front-of-package nutrition label that most influences the understanding of calories and important nutrients in processed food products in adult participants in Lima, Peru. Materials and Methods: A quasi-experimental cross-sectional study with 384 participant adults of both sexes. Participants were distributed into four equal groups: no nutritional labeling; daily food guide; stop sign warning labeling; and daily food guide with a traffic light symbol. Each participant was given a questionnaire with 10 pictures of food packaging with the labeling model that corresponded to their randomized group, along with 10 additional questions, for a total of 20 possible questionnaire points. Points were given for correct answers to the 20 questions on the questionnaire, such as identifying correctly the number of calories and/or critical nutrients. Results: The highest total scores were for the daily food guide-traffic light group (12.1 \pm 3.9$)$ and daily food guide group $(12.1 \pm 3.7)$, with significant differences with respect to the other two groups. There were also differences in the scores of the identification of calories, saturated fat, and total fat; the highest values were for the same two groups indicated above $(p<0.05)$. No differences in food labels were found for the identification of sugars or sodium. Conclusions: The daily food guide and daily food guide-traffic light models most influence the identification of calories and critical nutrients.

Keywords: Food labelling, product labeling, nutritional labeling, nutritional facts, nutritional information, nutritional value.

\section{INTRODUCCIÓN}

Dentro de las diez primeras causas de muerte en Perú, durante el 2014, se encontraron en el cuarto lugar las enfermedades del corazón, responsables del 9,6\% de las defunciones; en el noveno lugar, las enfermedades hipertensivas con el 3,9\%; y en el décimo lugar, la diabetes mellitus con el $3,8 \%$ (1). Se conoce que, dentro de las causas para desarrollar las enfermedades antes mencionadas, la presencia de sobrepeso y obesidad se asocian como los principales factores de riesgo, los cuales a su vez son el resultado de una alimentación desequilibrada y poca actividad física (2).

Una de las estrategias para disminuir la incidencia de estas enfermedades es la implementación de un modelo de etiquetado nutricional frontal, que pro- porciona información clara y simple a la población acerca del contenido nutricional de los alimentos industrializados, para contribuir al desarrollo de conductas alimentarias más saludables (3).

En la región latinoamérica, en el 2014, fue Ecuador el primer país que aprobó la implementación de un sistema de etiquetado tipo semáforo. En el año 2016 y con base en la evidencia desarrollada, Chile aprobó un etiquetado de advertencia octogonal frontal obligatorio (4), sistema que también adoptaron Uruguay y Perú, en este último país implementado en el 2019 y aprobado por el Ministerio de Salud del Perú mediante el Reglamento de la Ley N. ${ }^{\circ} 30021$, Ley de Promoción de la Alimentación Saludable (5).

Esta ley, que obliga a la industria de alimentos en Perú a la implementación del etiquetado de adver- 
tencia con el mensaje "alto en" en los productos que superan las cantidades máximas establecidas de azúcar, sodio, grasas saturadas o grasas trans (6) fue muy debatida, ya que las cantidades máximas permitidas por nutriente para rotular la advertencia se encontraban muy por encima de las recomendaciones de la Organización Panamericana de la Salud (OPS) (7).

Es entonces que, antes de ser aprobado el reglamento de la Ley N. ${ }^{\circ} 30021$, el Congreso del Perú propuso que la información referida al contenido de los nutrientes críticos y calorías en alimentos y bebidas se expresara en un gráfico similar a las Guías Diarias de Alimentación (GDA) con los colores del semáforo (8). Este etiquetado frontal señala la cantidad de calorías y determinados nutrientes, como la grasa total, las grasas saturadas, el sodio y los azúcares, que aporta una porción de alimento o bebida, e indica a través de los colores, rojo, amarillo y verde, el alto, medio o bajo contenido del nutriente crítico (9). A su vez, la Sociedad Nacional de Industrias del Perú también propuso un modelo de etiquetado nutricional, el GDA monocromo (9); sin embargo, ninguno de estos dos modelos se aprobó en Perú; y es el etiquetado de advertencia octogonal el que actualmente está vigente en este país desde el 17 de junio del 2019 (5).

Debido a que en el Perú se cuenta solo con estudios de percepción en relación con el etiquetado nutricional frontal $(10,11)$ y de influencia en la decisión de compra $(12,13)$, el objetivo de la presente investigación fue conocer el modelo de etiquetado nutricional que más influye en la capacidad de identificación del contenido de nutrientes críticos en productos industrializados (bebidas, cereales, galletas, grasas untables y snacks) en adultos de Lima, Perú.

\section{MATERIALES Y MÉTODOS}

\section{Diseño, población y muestra}

La investigación tuvo un diseño cuasiexperimental, de corte transversal. La población estuvo conformada por 5446794 adultos que residen en Lima Metropolitana, según el último Compendio Estadístico Poblacional de Lima (14), y quienes cumplieron con los siguientes criterios de inclusión: tener entre 18 años y 55 años, haber cursado como mínimo secundaria completa, tener residencia en Lima Metropolitana mayor a 12 meses y leer claramente. Asimismo, dentro de los criterios de exclusión estuvieron aquellos participantes con carreras pertenecientes a ciencias de la salud y de alimentos.

La muestra estuvo conformada por 384 adultos, quienes participaron voluntariamente y cumplieron con los criterios de inclusión. Para la selección de la muestra, se utilizó el muestreo probabilístico "aleatorio simple" (15) con un nivel de confianza del $95 \%$, heterogeneidad de $50 \%$ y un margen de error del $5 \%$.

\section{División de los participantes por grupo de eti- quetado frontal}

La muestra se dividió al azar en cuatro grupos de 96 participantes cada uno, a los que se le asignó un cuestionario con imágenes de etiquetado que presentaban variaciones:

- Sin modelo de etiquetado frontal (SM), grupo control.

- GDA: etiquetado conformado por las siglas de Guías Diarias de Alimentación, que señala la cantidad de energía, grasa total, grasas saturadas, sodio y azúcares que aporta una porción de un alimento o bebida, 
en relación con las necesidades diarias de una persona, en una dieta de $2000 \mathrm{kcal}$ (12).

- GDA-semáforo: representado por un GDA resaltado con los colores del semáforo, en relación con las necesidades diarias de una persona en una dieta de 2000 kcal: rojo (>25\% del valor diario), amarillo (10-25\% del valor diario) 0 verde $(<10 \%$ del valor diario). Este etiquetado evalúa energía, grasa total, grasas saturadas, sodio y azúcares que aporta una porción de alimento o bebida (12).

- Etiquetado de advertencia octogonal: octógono de color negro y blanco, con un mensaje de "alto en", que indica el contenido excesivo de sodio, azúcar total, grasas saturadas y grasas trans, en $100 \mathrm{~g} \mathrm{o} 100 \mathrm{ml}$ de producto (6).

Los modelos de etiquetado nutricional evaluados se presentan en la figura 1.

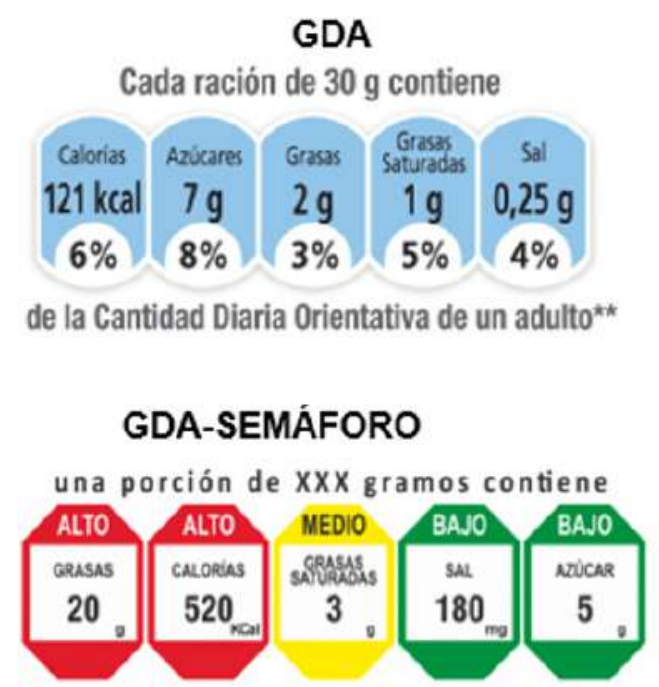

\section{Encuesta y cuestionario}

Esta encuesta se realizó antes de que se implementara el etiquetado de advertencia octogonal en Perú. A cada grupo se le aplicó un cuestionario, que fue sometido previamente a juicio de expertos y una prueba piloto para su validación.

El cuestionario constó de 10 figuras de empaques (dos figuras de bebidas, dos de cereales para el desayuno, dos de galletas, dos de grasas untables y dos de snacks salados) y 10 preguntas relacionadas con un nutriente (dos preguntas sobre azúcar, dos sobre sodio, dos sobre grasa saturada, tres sobre calorías y una sobre grasa total), estos alimentos fueron seleccionados tomando como referencia las estadísticas de consumo de la OPS (16), en las que se mencionan los alimentos ultraprocesados más consumidos en América Latina.

\section{ETIQUETADO DE ADVERTENCIA} OCTOGONAL

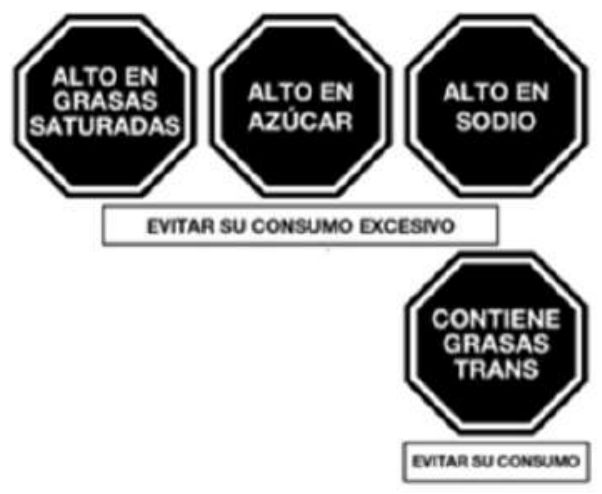

Figura 1. Modelos de etiquetado nutricional evaluados GDA, etiquetado de advertencia octogonal y GDA-semáforo.

Nota: el GDA muestra el contenido de calorías (kcal) y de nutrientes en $\mathrm{g} / \mathrm{mg}$, acompañados del porcentaje de valor diario (\% VD). El etiquetado de advertencia octogonal es un sello de advertencia negro y blanco, acompañado de la frase "alto en....", seguido del nutriente crítico que sobrepasa el límite establecido si es sólido o líquido. EI GDA-semáforo muestra la misma información del GDA, codificado por los colores verde, amarillo y rojo para alertar a los consumidores sobre los niveles bajos (<10\% VD), medios (10-25\% VD) y altos (>25\% VD) de los nutrientes, respectivamente. 
Cada pregunta evaluó la presencia elevada de un nutriente crítico al comparar dos empaques, ejemplo: ¿cuál de los productos de los empaques contiene más sodio? El participante marcó el producto $A \circ B$, según correspondía (Tabla 1).
En cada cuestionario se compararon empaques con un modelo de etiquetado nutricional frontal según el grupo asignado (GDA, GDA-semáforo, etiquetado de advertencia octogonal) frente a etiquetas sin etiquetado frontal (SM) (Figura 1).

Tabla 1. Perfiles de las informantes que conformaron la muestra estudiada

\begin{tabular}{|c|c|c|}
\hline Número & Preguntas & Respuestas \\
\hline 1 & Bebidas (¿Cuál de ellas contiene más calorías?) & \\
\hline a & Gaseosa & \\
\hline b & Bebida de durazno & \\
\hline 2 & Bebidas (¿Cuál de ellas contiene más azúcar?) & \\
\hline a & Gaseosa & \\
\hline b & Bebida de durazno & \\
\hline 3 & Cereales (¿Cuál de ellos contiene más sodio?) & \\
\hline a & Cereal sabor chocolate & \\
\hline b & Cereal a base de hojuelas de maíz & \\
\hline 4 & Cereales (¿Cuál de ellos contiene más calorías?) & \\
\hline a & Cereal sabor chocolate & \\
\hline b & Cereal a base de hojuelas de maíz & \\
\hline 5 & Galletas (¿Cuál de ellas contiene más azúcar?) & \\
\hline a & Galleta de chocolate con relleno de vainilla & \\
\hline b & Galleta de vainilla con relleno de chocolate & \\
\hline c & Ambas contienen la misma cantidad & \\
\hline 6 & Galletas (¿Cuál de ellas contiene más calorías?) & \\
\hline a & Galleta de chocolate con relleno de vainilla & \\
\hline b & Galleta de vainilla con relleno de chocolate & \\
\hline 7 & Grasas untables (¿Cuál de ellas contiene más grasas saturadas?) & \\
\hline a & Mantequilla & \\
\hline b & Margarina & \\
\hline 8 & Grasas untables (¿Cuál de ellas contiene más grasas totales?) & \\
\hline a & Mantequilla & \\
\hline b & Margarina & \\
\hline 9 & Snacks (¿Cuál de ellas contiene más grasas saturadas?) & \\
\hline a & Papas fritas & \\
\hline$b$ & Palitos de maíz & \\
\hline 10 & Snacks (¿Cuál de ellas contiene más sodio?) & \\
\hline a & Papas fritas & \\
\hline b & Palitos de maíz & \\
\hline
\end{tabular}

Fuente: basado en Roberto et al (21). 
Para el grupo SM, las imágenes del cuestionario no contenían ningún modelo de etiquetado en la parte frontal del empaque. En esta investigación, no se incluyó la tabla de información nutricional ya que no es obligatoria en Perú.

Los resultados obtenidos en las encuestas se contrastaron con las respuestas correctas para asignar el puntaje, ya fuera 002 puntos por cada respuesta acertada, lo que correspondía a un puntaje total del 0 a 20.

Los puntajes individuales se promediaron y se obtuvieron cuatro puntajes grupales.

\section{Análisis estadístico}

Los cálculos se realizaron en el programa SPSS v. 25 , previa prueba de normalidad. Para evaluar si existía diferencia estadísticamente significativa en los puntajes sobre la capacidad de identificar la presencia elevada de nutrientes críticos entre los grupos: SM, GDA, etiquetado de advertencia octogonal y GDA-semáforo, se utilizó el análisis de varianza (ANOVA) (15).

Adicionalmente, para comparar los puntajes obtenidos en la identificación de calorías, azúcar, sodio, grasas totales y grasa saturada, los grupos SM, GDA, etiquetado de advertencia octogonal y GDA-semáforo entre sí, se aplicó la prueba post hoc de Tukey (15) y para analizar si existía diferencia en los resultados según el sexo, profesión y edad por grupos, se utilizó la prueba ji al cuadrado de Pearson (15).

\section{Consideraciones éticas}

La investigación fue aprobada por el Comité de Ética de la Universidad Femenina del Sagrado Corazón (UNIFÉ) y los participantes firmaron un consentimiento informado.

\section{RESULTADOS}

Respecto a la caracterización de la muestra que se detalla en la tabla 2, se observa que el $51 \%$ estuvo conformada por mujeres y el $49 \%$ por hombres; sin embargo, existieron diferencias porcentuales al analizar el sexo por grupos. En relación con la ocupación o profesión del total de la muestra, $22 \%$ fueron administradores, seguido de estudiantes universitarios de diversas ramas con $18 \%$, ingenieros de diversas especialidades con un $14 \%$, docentes $11 \%$, militares $5 \%$ y otras profesiones u ocupaciones $24 \%$. Respecto a la edad, la mayoría de la muestra (56 \%) tuvo entre 18 y 25 años. Cabe mencionar que las características de los participantes por grupos (sexo, profesión y edad) no influyeron en los resultados, según la prueba ji al cuadrado de Pearson.

Entre los cuatro grupos estudiados, se presentaron diferencias significativas entre los puntajes para identificar calorías, grasas saturadas y grasa total, lo mismo que en el puntaje total (ANOVA $<0,05)$, pero no en aquellos de la identificación de azúcar y sodio. Los mayores puntajes totales fueron para los grupos GDA-semáforo $(12,1 \pm 3,9)$ y $\operatorname{GDA}(12,1 \pm 3,7)$ con diferencias significativas respecto a los otros dos grupos (ANOVA=0,000) (Tabla 3).

El grupo que presentó el mayor puntaje en la identificación de grasas saturadas fue el GDAsemáforo $(2,8 \pm 1,3)$ con diferencias significativas frente a los otros tres, según la prueba de comparación múltiple de Tukey; en cuanto a la identificación de la grasa total, el mayor puntaje fue para el grupo GDA $(1,1 \pm 1,0)$ y el menor, para el octágono $(0,7 \pm 0,9)$, con diferencias estadísticas entre ellos y respecto a los otros dos grupos (Turkey<0,05) (Tabla 3). 
Tabla 2. Caracterización de la muestra

\begin{tabular}{|c|c|c|c|c|c|c|c|c|c|c|c|}
\hline \multirow[t]{3}{*}{ Variable } & \multirow{2}{*}{\multicolumn{2}{|c|}{$\begin{array}{c}\text { Total } \\
(n=384)\end{array}$}} & \multirow{2}{*}{\multicolumn{2}{|c|}{$\begin{array}{c}\text { SM } \\
(n=96)\end{array}$}} & \multirow{2}{*}{\multicolumn{2}{|c|}{$\begin{array}{c}\text { GDA } \\
(n=96)\end{array}$}} & \multirow{2}{*}{\multicolumn{2}{|c|}{$\begin{array}{l}\text { Etiquetado de } \\
\text { advertencia octogonal } \\
(n=96)\end{array}$}} & \multirow{2}{*}{\multicolumn{2}{|c|}{$\begin{array}{c}\text { GDA-semáforo } \\
(n=96)\end{array}$}} & \multirow[t]{3}{*}{ Valor de $\mathrm{p}^{*}$} \\
\hline & & & & & & & & & & & \\
\hline & $n$ & $\%$ & $\mathrm{n}$ & $\%$ & $n$ & $\%$ & $n$ & $\%$ & $n$ & $\%$ & \\
\hline \multicolumn{12}{|l|}{ Sexo } \\
\hline Masculino & 187 & 49 & 63 & 66 & 37 & 39 & 50 & 52 & 37 & 39 & \multirow{2}{*}{0,854} \\
\hline Femenino & 197 & 51 & 33 & 34 & 59 & 61 & 46 & 48 & 59 & 61 & \\
\hline \multicolumn{12}{|l|}{ Profesión } \\
\hline Administrador & 86 & 22 & 22 & 23 & 30 & 31 & 17 & 18 & 17 & 18 & \multirow{7}{*}{0,300} \\
\hline Comunicador & 22 & 6 & 4 & 4 & 2 & 2 & 8 & 8 & 8 & 8 & \\
\hline Docente & 40 & 11 & 2 & 2 & 17 & 18 & 7 & 7 & 14 & 15 & \\
\hline Universitario & 70 & 18 & 20 & 21 & 11 & 11 & 23 & 24 & 16 & 17 & \\
\hline Ingeniero & 52 & 14 & 16 & 17 & 15 & 16 & 10 & 10 & 11 & 11 & \\
\hline Militar & 21 & 5 & 13 & 13 & 0 & 0 & 5 & 6 & 3 & 3 & \\
\hline Otros & 93 & 24 & 19 & 20 & 21 & 22 & 26 & 27 & 27 & 28 & \\
\hline \multicolumn{12}{|l|}{ Edades } \\
\hline $18-25$ años & 214 & 56 & 59 & 62 & 36 & 37 & 62 & 65 & 57 & 59 & \multirow{4}{*}{1,000} \\
\hline 26-35 años & 83 & 22 & 29 & 30 & 20 & 21 & 16 & 17 & 18 & 19 & \\
\hline 36-45 años & 51 & 13 & 6 & 6 & 22 & 23 & 9 & 9 & 14 & 15 & \\
\hline 46-55 años & 36 & 9 & 2 & 2 & 18 & 19 & 9 & 9 & 7 & 7 & \\
\hline
\end{tabular}

$\mathrm{SM}=$ sin modelo de etiquetado nutricional

$\mathrm{GDA}=$ guía diaria de alimentación

GDA-semáforo= guía diaria de alimentación-semáforo

$\mathrm{X} \pm \mathrm{DE}=$ promedio \pm desviación estándar

* valor de p como resultado de la prueba ji al cuadrado de Pearson, comparando la distribución entre los cuatro grupos según modelo de etiquetado y variable.

Tabla 3. Comparación de los puntajes obtenidos de la identificación de calorías, nutrientes críticos y en la encuesta total, según los grupos estudiados

\begin{tabular}{lccccc}
\hline Variable & SM & GDA & Octágono & GDA-semáforo & Valor de $\mathbf{p}^{*}$ \\
\hline & $(\mathrm{n}=96)$ & $(\mathrm{n}=96)$ & $(\mathrm{n}=96)$ & $(\mathrm{n}=96)$ & \\
& $\mathrm{X} \pm \mathrm{DE}$ & $\mathrm{X} \pm \mathrm{DE}$ & $\mathrm{X} \pm \mathrm{DE}$ & $\mathrm{X} \pm \mathrm{DE}$ & \\
\hline Calorías & $2,8 \pm 1,5^{\mathrm{a}}$ & $3,5 \pm 1,8^{\mathrm{b}}$ & $2,5 \pm 1,4^{\mathrm{a}}$ & $3,1 \pm 1,9^{\mathrm{c}}$ & 0,002 \\
Azúcar & $2,6 \pm 1,3$ & $2,9 \pm 1,1$ & $2,9 \pm 1,3$ & $2,9 \pm 1,3$ & 0,279 \\
Sodio & $2,2 \pm 1,2$ & $2,3 \pm 1,2$ & $2,3 \pm 1,3$ & $2,3 \pm 1,0$ & 0,858 \\
Grasas saturadas & $2,1 \pm 1,3^{\mathrm{a}}$ & $2,3 \pm 1,4^{\mathrm{a}}$ & $2,1 \pm 1,3^{\mathrm{a}}$ & $2,8 \pm 1,3^{\mathrm{b}}$ & 0,002 \\
Grasa total & $0,9 \pm 0,9^{\mathrm{a}}$ & $1,1 \pm 1,0^{\mathrm{b}}$ & $0,7 \pm 0,9^{\mathrm{c}}$ & $1,0 \pm 1,0^{\mathrm{a}}$ & 0,033 \\
Puntaje total & $10,6 \pm 3,1^{\mathrm{a}}$ & $12,1 \pm 3,7^{\mathrm{b}}$ & $10,5 \pm 2,9^{\mathrm{a}}$ & $12,1 \pm 3,9^{\mathrm{b}}$ & 0,000 \\
\hline
\end{tabular}

$\mathrm{SM}=$ sin modelo de etiquetado nutricional

$\mathrm{GDA}=$ guía diaria de alimentación

GDA-semáforo= guía diaria de alimentación-semáforo

$\mathrm{X} \pm \mathrm{DE}=$ promedio \pm desviación estándar

*Valor de $p=$ diferencia entre los grupos según la prueba de ANOVA

$a, b$ y $c=$ las letras distintas en cada columna indican los grupos en los que hubo diferencias significativas, según la prueba de Tukey $(p<0,05)$. 


\section{DISCUSIÓN}

Los principales resultados del presente estudio fueron que, entre los cuatro grupos estudiados, uno sin etiquetado frontal (SM) y tres modelos de etiquetado nutricional frontal: GDA, Etiquetado de advertencia octogonal y GDA-semáforo, se encontraron diferencias estadísticas significativas en los puntajes de la identificación de calorías, grasas saturada y grasa total, pero no en aquellos de la identificación de azúcar y sodio. Los mayores puntajes totales fueron para los grupos GDA-semáforo $(12,1 \pm 3,9)$ y $\operatorname{GDA}(12,1 \pm 3,7)$. El grupo que presentó el mayor puntaje en la identificación de grasas saturadas fue el GDA-semáforo $(2,8 \pm 1,3)$, con diferencias significativas frente a los otros tres. Por último, en cuanto a la identificación de la grasa total, el mayor puntaje fue para el grupo GDA $(1,1 \pm 1,0)$.

Como se observa, el GDA-semáforo fue uno de los modelos de etiquetado frontal que mejor permitió la identificación del aporte calórico y de nutrientes críticos en el presente estudio, distinto a lo hallado por Khandpur et al. (17) en el año 2018, quienes concluyeron que el sello de advertencia (símil al etiquetado de advertencia octogonal) fue el mejor modelo de etiquetado nutricional frente al etiquetado GDA-semáforo, para ayudar a los consumidores a identificar los nutrientes de interés y cambiar las intenciones de compra en el contexto brasileño. Si bien es cierto que los resultados de la investigación mencionada no coinciden con los hallados en el presente estudio, al evaluar el diseño de los sellos de advertencia propuestos en la investigación brasileña se puede observar que estos ocupan aproximadamente el $20 \%$ del área frontal del empaque, en comparación del GDA-semáforo, que solo ocupa el $6 \%$ del área frontal del empaque, lo que indica que la diferencia de los resultados puede deberse a que la advertencia genera más impacto visual al ser más grande, de color negro y con un único fondo blanco.

En otro estudio, Crosetto et al. (18) encontraron que al evaluar el modelo GDA frente al GDA-semáforo, el GDA obtiene un mejor puntaje que el modelo GDA-semáforo cuando los participantes no tienen un tiempo limitado para el análisis del etiquetado; por el contrario, cuando el tiempo es limitado, ambos modelos tienen una eficacia idéntica al evaluar cuatro nutrientes críticos, incluso el GDA-semáforo supera el GDA y evalúa siete nutrientes críticos correctamente. Estos resultados se asemejan a lo hallado en la presente investigación, ya que tanto el GDA como el GDAsemáforo lograron obtener la misma media.

Siegrist et al. (19), quienes examinaron la efectividad de tres formatos diferentes, Tabla de Información Nutricional, el GDA y el GDA-semáforo, encontraron que los participantes necesitaron más tiempo para procesar el formato GDA en comparación con el formato del GDA-semáforo y el formato de la Tabla de Información Nutricional. Con respecto al procesamiento de la información, el modelo de etiquetado GDA-semáforo fue mejor que los otros dos, debido a que, según los autores, se presenta como una forma amigable de comunicar información nutricional para el consumidor. Estos resultados concuerdan parcialmente con los hallados en la presente investigación, ya que tanto el GDA-semáforo $(12,1 \pm 3,9)$ como el GDA $(12,1 \pm 3,7)$ obtuvieron la misma media. Maubach et al. (20) también encontraron que el modelo de etiquetado semáforo tuvo un impacto significativo al evaluar el perfil nutricional de los alimentos frente el etiquetado Stars rating, el GDA y el grupo control (sin etiquetado nutricional frontal).

Roberto et al. (21), en el año 2012 en Reino Unido, hallaron que el modelo de etiquetado Traffic 
Light+ (semáforo) obtuvo un mejor puntaje que el modelo Facts Up Front (GDA) sobre la capacidad de conocimiento de nutrición y percepciones de la etiqueta. Además, al ver el detallado por nutrientes, ellos mencionan que en su investigación el grupo control no difirió del grupo Facts Up Front en el cuestionario de grasas saturadas, resultado que coincide con lo hallado en la presente investigación, en la que no se encontró diferencia significativa $(p=0,139)$ entre el grupo SM y el grupo GDA para los resultados de grasa saturada. Roberto et al. (21) también mencionan que el grupo control no difirió del grupo Facts Up Fronten el cuestionario de azúcar, resultado bastante similar a lo hallado en la presente investigación, ya que no se encontró diferencia significativa $(p=0,279)$ entre el grupo SM y los grupos GDA, etiquetado de advertencia octogonal y GDA-semáforo al evaluar este nutriente.

Otra investigación que detalla la evaluación por nutrientes fue la realizada por Ducrot et al. (22), en la cual se menciona que ni el modelo de etiquetado GDA ni el modelo de etiquetado GDA-semáforo influyeron al evaluar el contenido de grasas totales y sodio, al igual que la presente investigación; sin embargo, difiere en el caso de grasas saturadas para el GDA-semáforo, ya que se encontró que sí influye en la identificación de este nutriente crítico con un $p=0,01$; a diferencia de la investigación de Ducrot.

En la investigación de Ducrot et al. (22) también se menciona que el impacto de los diferentes modelos de etiquetado fue similar en todos los subgrupos sociodemográficos. Esta investigación respalda lo referido por la población peruana en el estudio realizado en febrero del 2018 en 17 departamentos del Perú por la Compañía Peruana de Estudios de Mercado y Opinión S.A.C (11), en el que consultaron a los consumidores qué modelo de etiquetado nutricional preferían; el $67 \%$ refirió que el semáforo.
Si bien fue un estudio de percepción, lo hallado en la presente investigación contribuye a lo encontrado por la compañía encuestadora, ya que, además de ser el GDA-semáforo el etiquetado frontal que el consumidor prefiere ver en los empaques para la elección de alimentos saludables, también es el que logra entenderse mejor frente a los otros modelos de etiquetado nutricional.

En relación con el modelo preferido por los peruanos, la elección probablemente se debe a que la codificación de colores aumenta la capacidad de percepción de los consumidores a tomar decisiones saludables, además, que va acompañado de texto que describe el contenido bajo, medio 0 alto de cada nutriente (23). Esto fue demostrado en la investigación realizada por Aschemann-Witzela (23) en mil consumidores alemanes y polacos, en la que evaluaron diferentes modelos de etiquetado nutricional y encontraron que los formatos de codificación de color y texto, particularmente la codificación de colores en Alemania, aumentaron la salubridad de las opciones de productos cuando se les pidió a los consumidores elegir un producto saludable, pero no cuando se les pidió que eligieran según sus preferencias; es decir, si bien los consumidores saben distinguir el contenido de nutrientes críticos según el modelo de etiquetado, estos modelos no influyen en la motivación de los consumidores para elegir alimentos saludables.

En Ecuador, país que tiene el etiquetado tipo semáforo implementado actualmente, Poveda (24) realizó una investigación a través de entrevistas a profundidad a padres y madres de familia para evaluar el impacto del sistema GDA-semáforo en el etiquetado. El investigador halló que aunque los participantes aceptaron que continuaban comprando los mismos productos y marcas, sí hubo reducción en el consumo de aquellos productos con alto contenido de azúcar, sal y grasas; lo que demostraría que de alguna manera este modelo 


\section{Comparación de tres modelos de etiquetado}

de etiquetado está generando una modificación en la conducta alimentaria.

Finalmente, de acuerdo con los resultados obtenidos en la presente investigación, se concluye que existen diferencias en la identificación del contenido de nutrientes críticos de productos industrializados en adultos de Lima, Perú. Los modelos de etiquetado nutricional GDA y GDA-semáforo son los que más influyen en la población encuestada en este estudio. Sin embargo, es importante considerar que ninguna estrategia para el etiquetado será suficiente para lograr modificar los hábitos alimentarios, esta debe ir acompañada de una adecuada educación alimentaria y nutricional (25).

\section{CONFLICTO DE INTERESES}

Los autores declaran no tener ningún conflicto de interés.

\section{-Referencias}

1. Perú, Ministerio de Salud .Oficina General de Tecnología de la Información; Lima; 2014. Minsa.gob.pe. [Internet]. [Citado 18 de septiembre 2017] Disponible en: http://www.minsa.gob.pe/estadisticas/ estadisticas/mortalidad/macros.asp?00

2. Adman CS, Márcio FM, Roberto WJ, Maria LZ, Paulo CA, Marta MC. Factores de riesgo para Diabetes Mellitus Tipo 2 en universitarios: asociación con variables sociodemográficas. Rev. Latino-Am. Enfermagem. 2014;22(3):484-90. Disponible en: http://www.scielo.br/pdf/rlae/v22n3/es_0104-1169-rlae-22-03-00484.pdf

3. FAO y OPS. Preguntas y respuestas sobre la ley de alimentos. 2018. [Internet]. [Citado julio 2020] Disponible en: http://www.fao.org/3/ca1314es/ca1314es.pdf

4. OPS. Avanza el etiquetado frontal en las Américas. [Citado julio de 2020]. Disponible en: https://www.paho.org/hq/index. php?option=com_content\&view=article\&id=14541:avanza-el-etiquetado-frontal-en-las-americas\&ltemid=4327\&lang=es

5. Perú, Ministerio de Salud. Decreto Supremo que aprueba el Reglamento de la Ley № 30021, Ley de Promoción de la Alimentación Saludable. [Citado noviembre de 2017]. Disponible en: http://www2.congreso.gob.pe/sicr/cendocbib/con4_uibd.nst/52 89E04A2A160ABD052581A10070E6CE/\$FILE/2_decreto_supre_017_de_alimentacion.pdf

6. Perú, Ministerio de Salud. Manual de advertencias publicitarias del reglamento de la ley no. 30021, ley de promoción de la alimentación saludable para niños, niñas y adolescentes. [Internet]. [Citado noviembre de 2017]. Disponible en: http://www.puntofocal.gov.ar/notific_otros_miembros/per97_t.pdf

7. OPS. Modelo de perfil de nutrientes de la Organización Panamericana de la Salud. 2016. [Internet]. [Citado julio de 2020]. Disponible en: https://iris.paho.org/handle/10665.2/18622

8. Peru21.pe [Internet]. Lima: Grupo El Comercio. [actualizado 14 de junio del 2017; citado 05 de enero 2019]. Disponible en: https://peru21.pe/economia/sociedad-nacional-industrias-rechaza-semaforo-nutricional-propuesto-daniel-salaverry-81282

9. México, Gob.mx. México: Gob.mx. 2016. [Internet]. [Citado octubre de 2017]. Disponible en: https://www.gob.mx/salud/articulos/sabias-que-el-distintivo-nutrimental-es-voluntario

10. Valverde-Aguilar M, Espadín-Alemán CC, Torres-Ramos NE, Liria-Domínguez R. Preferencia de etiquetado nutricional frontal: etiquetado de advertencia octogonal frente a semáforo GDA en mercados de Lima, Perú. Acta Méd Peru. 2018; 35(3):145-52. Disponible en: http://www.scielo.org.pe/scielo.php?script=sci_arttext\&pid=S1728-59172018000300002\&lng=es

11. Compañía peruana de estudios de mercado y opinión S.A.C. Perú urbano. etiquetado nutricional en alimentos y bebidas envasadas. [Internet]. [Citado octubre de 2018]; Disponible en: https://www.cpi.pe/images/upload/paginaweb/archivo/23/opnac_etiqueta_saludable_peru_201803.pdf 
12. Ezcurra DE, Fernandez ED, Henostroza RS. Diseño cuasi experimental para el análisis de la decisión de compra del consumidor limeño ante los sistemas de rotulado frontal en abarrotes y lácteos. Pontificia Universidad Católica Del Perú Facultad de Gestión y Alta Dirección. Lima: 2019. [Internet]. [Citado noviembre de 2019] Disponible en: http://tesis.pucp.edu.pe/repositorio/bitstream/handle/20.500.12404/15570/EZCURRA_VILLENA_FERN\%c3\%81NDEZ_LLATAS_HENOSTROZA_MENDOZA.pdf?sequence=18isAllowed=y

13. Bernales A. Uso del etiquetado nutricional en la decisión de compra de alimentos en consumidores de un supermercado peruano y otro ecuatoriano. Universidad Nacional Mayor de San Marcos Perú, Facultad de Medicina, Escuela Profesional de Nutrición. Lima: 2018. [Internet]. [Citado enero 2019]. Disponible en: https://cybertesis.unmsm.edu.pe/bitstream/handle/20.500.12672/9400/Bernales_ca.pdf?sequence=3\&isAllowed=y

14. Perú INEI. Provincia de Lima Compendio Estadístico. Lima: Instituto Nacional de Estadística e Informática; 2017. [Citado enero de 2019]. Disponible en: https://www.inei.gob.pe/media/MenuRecursivo/publicaciones_digitales/Est/Lib1477/libro.pdf

15. Hernández-Sampieri. Metodología de la Investigación: ¿Cómo seleccionar una muestra? México D.F: McGraw Hill; 2014

16. Organización Panamericana de la Salud. Alimentos y bebidas ultraprocesados en América Latina: ventas, fuentes, perfiles de nutrientes e implicaciones. Washington, D.C: OPS; 2019.

17. Khandpur N, Sato PDM, Mais LA, Martins APB, Spinillo CG, Garcia T et al. Are Front-of-Package Warning Labels More Effective at Communicating Nutrition Information than Traffic-Light Labels? A Randomized Controlled Experiment in a Brazilian Sample. Nutrients. 2018;10(6): 688. Disponible en: http://www.mdpi.com/2072-6643/10/6/688

18. Crosetto P, Muller L, Ruffieux B. Helping consumers with a front-of-pack label: Numbers or colors?: Experimental comparison between Guideline Daily Amount and Traffic Light in a diet-building exercise. J Econ Psychol. 2016;55:30-50. https://doi.org/10.1016/j.joep.2016.03.006

19. Siegrist M, Leins-Hess R. y Keller $C$. Which front-of-pack nutrition label is the most efficient one? The results of an eye-tracker study. Food Qual Prefer. 2015;39:183-90. https://doi.org/10.1016/j.foodqual.2014.07.010

20. Maubach N, Hoek J, Mather D. Interpretive front-of-pack nutrition labels. Comparing competing recommendations. Appetite. 2014;82(1):67-77. https://doi.org/10.1016/j.appet.2014.07.006

21. Roberto CA, Bragg MA, Schwartz MB, Seamans MJ, Musicus A, Novak N, Brownell KD. Facts up front versus traffic light food labels: A randomized controlled trial. Am J Prev Med. 2012;43(2):134-41. Disponible en: https://www.ncbi.nlm.nih.gov/pubmed/22813677

22. Ducrot P, Chantal J, Méjean C, Kesse-Guyot E, Touvier M, Fezeu L, Hercberg S y et al. Impact of Different Front-of-Pack Nutrition Labels on Consumer Purchasing Intentions: A Randomized Controlled Trial. American Journal of Preventive Medicine. 2016;50(5):627-36. https://doi.org/10.1016/j.amepre.2015.10.020

23. Aschemann-Witzel J, Grunert KG, van Trijp HCM, Bialkova S, Raats MM, Hodgkins C, et al. Effects of nutrition label format and product assortment on the healthfulness of food choice. Appetite. [Internet]. 2013. [Citado 3 de octubre 2018]; 71(1): 63-74. https://doi.org/10.1016/j.appet.2013.07.004

24. Poveda A. Impacto del sistema de alertas "semáforo" de contenido de azúcar, sal y grasa en etiquetas de alimentos procesados: enfoque cualitativo. Revista de Ciencias Sociales y Humanas de la Universidad Politécnica Salesiana del Ecuador. 2016; XIV (25):48-60. http://doi.org/10.17163/uni.n25.2016.03

25. Centers for Desease Control and Prevention. Strategies to Prevent Obesity. 2019. [Citado 22 de julio 2020]. Disponible en: https://www.cdc.gov/obesity/strategies/index.html 